\title{
Técnicas de Aprendizado de Máquina Aplicadas na Previsão de Produtividade de Operadores de Centros de Teleatendimento
}

\author{
Evandro L. Oliveira* José M. Torres ${ }^{* *}$ Rui S. Moreira ${ }^{* * *}$ \\ Rafael A. F. Lima**** \\ * ISUS Unit, FCT, Universidade Fernando Pessoa, Porto, Portugal \\ (e-mail: evandro.oliveira@ufp.edu.pt http://isus.ufp.pt) \\ ** ISUS Unit, FCT, Universidade Fernando Pessoa, LIACC, FEUP, \\ Universidade do Porto, Porto, Portugal (e-mail: jtorres@ufp.edu.pt \\ http://isus.ufp.pt) \\ *** ISUS Unit, FCT, Universidade Fernando Pessoa, LIACC, FEUP, \\ INESC TEC, Porto, Portugal (e-mail: rmoreira@ufp.edu.pt \\ http://isus.ufp.pt) \\ **** Universidade Federal de Minas Gerais, Belo Horizonte, Brasil \\ (e-mail: rafaelfrancalima@gmail.com)
}

\begin{abstract}
Improving productivity is a major challenge faced by companies with large numbers of employees. Therefore, the existence of productivity prediction tools are an important mechanisms for the strategic planning of laborer intensive companies. This paper focuses on the use of machine learning technologies to predict the productivity of tele-service workers. More preciselly, some forecast models have been adjusted and tested with 370 features exgracted from a population of approximately 15,000 employees. This population was taken from the workforce of a large Brazilian tele-service provider. The features were extracted from the profiles of workers and then filtered through the use of corelation and selection of variables. The selected features were used for comparing the productivity prevision obtained from several machine learning algorithms (RF - Random Forest, MLP - Perceptron Multicamadas, NB - Naive Bayes, XGBoost - Extreme Gradient Impulse and DMM - Dummy). The hyper parameterization of ML models was also considered on the comparison of the most suited algorithms for the prediction problem. The hyper parameterization, as well as the feature selection, were adjusted through the use of an evolutionary algorithm and allowed obtaining promising prediction results.

Resumo: Melhorar a produtividade é um grande desafio enfrentado por companhias com um grande número de funcionários. Portanto, a existência de ferramentas de previsão de produtividade são mecanismos importante para o planejamento estratégico das empresas intensamente dependentes do trabalho dos funcionários. Este artigo foca o uso de tecnologias de aprendizado de máquina para prever a produtividade dos trabalhadores no serviço de teleatendimento. Mais precisamente, neste trabalho foram ajustados e testados vários modelos de previsão com 370 variáveis extraídas de uma população de aproximadamente 15 mil funcionários. Esta população alvo foi amostrada a partir da força de trabalho de uma grande empresa de prestação de serviços de teleatendimento brasileira. As variáveis foram extraídas do perfil dos agentes de teleatendimento e, em seguida, filtradas por processos de correlação e seleção de variáveis. As variáveis selecionadas foram utilizadas para comparar a previsão de produtividade obtida através de vários algoritmos de aprendizado máquina (cf. RF - Random Forest, MLP - Multilayer Perceptron, NB - Naive Bayes, XGBoost - Extreme Gradient Boosting e DMM Dummy). A hiper parametrização destes modelos de ML foi também considerada na comparação dos algoritmos mais adequados para o problema de previsão. As hiper parametrizações, assim como a seleção de variáveis, foram ajustadas através do uso de um algoritmo evolutivo e permitiram obter resultados de previsão bastante promissores.
\end{abstract}


Keywords: call center, feature selection, machine learning, classification models.

Palavras-chaves: teleatendimento, seleção de variáveis, aprendizado de máquina, modelos de classificação.

\section{INTRODUÇÃO}

O setor de teleatendimento se destaca no mercado econômico por oferecer serviços de atendimento primários entre os clientes e as suas empresas fornecedoras. Estes serviços empregam milhares de profissionais em todo o mundo Silva (2007). Um dos problemas enfrentados pelas empresas de teleatendimento é a gestão da produtividade dos seus colaboradores.

As empresas de teleatendimento buscam melhorar sua gestão econômica com estudos a respeito da produtividade de seus funcionários. Contudo, entender as reais causas que influenciam na produtivo dos profissionais é um trabalho complexo, visto que dependendo do número de características disponíveis dos funcionários, é difícil traçar um perfil de produtividade Ávila Assunção and de Oliveira Vilela (2003). Consideramos que este é um problema com potencialidade para ser abordado através da utilização de ferramentas de aprendizado de máquina. Nesse trabalho aplica-se a arquitetura de algoritmos de aprendizado apresentada na Figura 1, combinada com o ajuste da hiper-parametrização e a seleção de variáveis de entrada dos modelos, com o objetivo de comparação para obtenção da melhor previsão para o problema de produtividade.

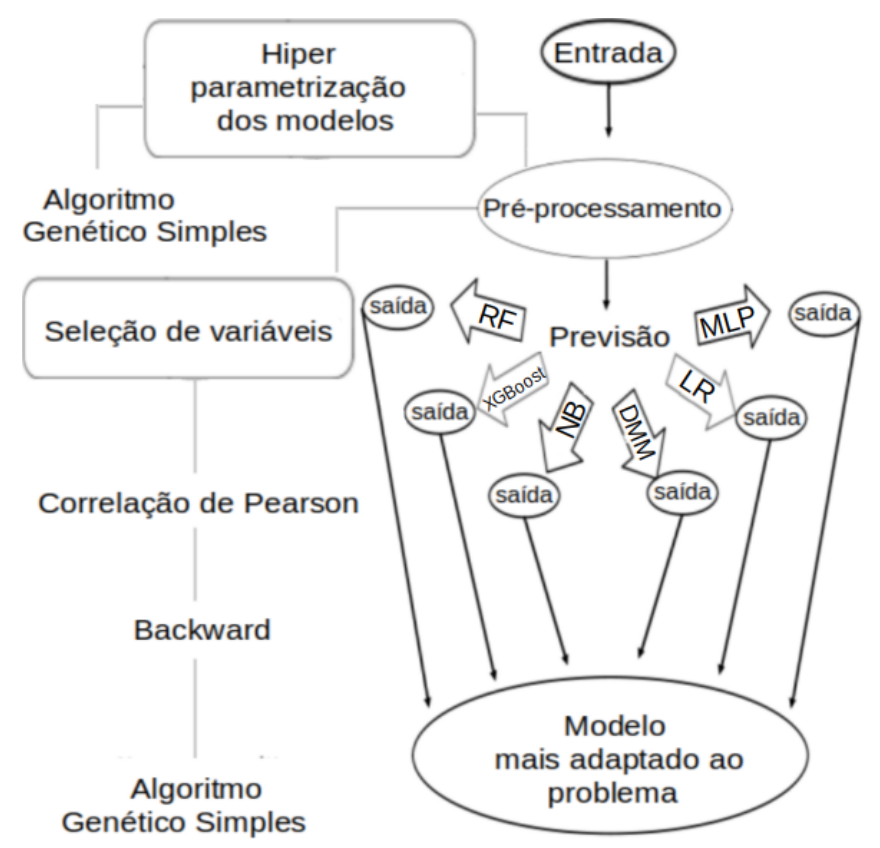

Figura 1. Arquitetura de previsão para escolha do melhor modelo de previsão

Foram coligidas 370 variáveis da informação disponível no perfil dos funcionários da empresa. Essas variáveis foram posteriormente transformadas através de um processo de normalização e binarização. O pre-processamento dos dados de entrada dos modelos considerou ainda a criação e organização de variáveis em função da periodicidade e do tempo, utilizando a técnica Nested Cross Validation Cochrane (2018).

Antes de serem submetidas aos modelos, as variáveis foram ordenadas e selecionadas em função do seu factor de correlação de Pearson com a variável de saída (cf. produtividade). Além de avaliar essa correlação, foi posteriormente efetuada uma avaliação dos melhores resultados de predição conforme a combinação dessas variáveis. Esta avaliação foi realizada comparando os resultados de predição em função das combinações de variáveis de entrada dos modelos, selecionadas pela técnica de backward e por utilização de um algoritmo genético.

Para além do estudo das melhores combinações de variáveis de entrada dos modelos de previsão, foram ainda consideradas várias hiper parametrizações dos modelos testados, para encontrar a configuração mais adequada à previsão de produtividade. A estratégia escolhida para tuning dos hiper-parâmetros dos modelos recorreu também a um algoritmo genético, considerando todas as variáveis como entrada sem combinações.

A avaliação da previsão de produtividade foi aplicada nas unidades de atendimento por telefone da empresa alvo da pesquisa, considerando dados recolhidos pela organização num universo que excedeu os 15 mil colaboradores (todos operadores de teleatendimento). Os dados foram recolhidos pela empresa no decurso normal do seu funcionamento interno, cumprindo os preceitos legais, éticos e respectivo consentimento informado dos seus colaboradores.

O artigo está organizado da seguinte forma: a secção 2 descreve o ambiente e explica as regras que envolvem o problema; A seç̧ão 3 detalha a organização e divisão da informação para entrada nos modelos de previsão; A secção 4 apresenta os modelos; A secção 5 apresenta a estrutura do algoritmo genético para tuning dos modelos e seleção de variáveis; A secção 6 demonstra como foi efetuado o processo de tuning dos modelos; Na secção 7, são apresentadas as estratégias de seleção de variáveis; Na secção 8, é detalhado o processo de previsão e resultados; e na secção ??, apresentam-se os resultados e conclusões.

\section{CENÁRIO E EXPLANAÇÃO DO PROBLEMA}

Os 15.621 funcionários alvos da pesquisa trabalham numa companhia de grande porte que se foca na terceirização de serviços nas áreas de teleatendimento, consultoria, software e gestão em saúde. Esta companhia atua em todo o território brasileiro, incorporando portanto colaboradores em diferentes regiões do país.

O trabalho desenvolvido pelos operadores de teleatendimento desta companhia consiste no esclarecimento de dúvidas dos clientes, reclamações, compra de serviços e resolução de problemas de caráter financeiro específicos das empresas contratantes que terceirizam o seu teleatendimento. 
A produtividade do trabalho prestado pelos operadores é definida por meio de indicadores de desempenho. Esses indicadores consideram, por exemplo, o tempo médio que as ligações de atendimento ao cliente duram, o número de re-chamadas, o número de pausas do funcionário, etc. Quando os indicadores são consolidados, um funcionário é considerado produtivo pela empresa caso ele atinja pelo menos $55 \%$ do total que ele poderia atingir (percentual de atingimento definido pela empresa, individualmente para cada trabalhador). O desafio é portanto prever se cada um dos funcionários manterá, descerá ou aumentará o seu desempenho no prazo de uma semana.

Busca-se, com esse trabalho, prever a possibilidade dos operadores de teleatendimento serem ou não produtivos na semana seguinte à previsão. A previsão de produtividade dos funcionários para a próxima semana é obtida a partir de variáveis/características dos funcionários relativas às semanas anteriores. Este tipo de informação é importante para empresa na tomada de decisões relativas à sua gestão e promoção da produtividade.

\section{PROCESSAMENTO DE INFORMAÇÕES}

A abordagem adotada neste trabalho consiste na comparação de vários modelos de aprendizado máquina com potencial de previsão da produtividade. Esta abordagem foi seguida com caráter experimental e adaptado-se às particularidades do problema estudado. Nesta adaptação, os dados foram organizados e transformados para se coadunarem como entrada aos modelos selecionados.

\subsection{Organização das variáveis de entrada}

Disposição das variáveis Para simplificar a explanação dos dados e a metodologia seguida neste trabalho, vamos considerar que, hipoteticamente, a empresa possuía apenas 3 colaboradores com 10 variáveis cada um (ver Figura 2). Os 3 colaboradores nessa figura são xxx, yyy e zzz e suas características/variáveis são: sexo, casado, P1, P2, P3, P4, P5, P6, P7 e P8.

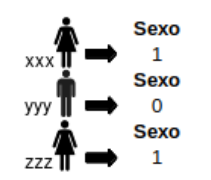

$\begin{array}{ccc}\text { Casado } & \mathbf{P 1} & \mathbf{P} \\ 1 & 0,44 & 0,58 \\ \text { Casado } & \mathbf{P 1} & \mathbf{P} \\ 1 & 0,35 & 0,89 \\ \text { Casado } & \mathbf{P 1} & \mathbf{P} \\ 0 & 0,12 & 0,25\end{array}$

$\begin{array}{cccc}\text { P2 } & \text { P3 } & \text { P4 } & \text { P5 } \\ 0,58 & 0,68 & 0,45 & 0,74 \\ \text { P2 } & \text { P3 } & \text { P4 } & \text { P5 } \\ 0,89 & 0,78 & 0,87 & 0,81 \\ \text { P2 } & \text { P3 } & \text { P4 } & \text { P5 } \\ 0,25 & 0,44 & 0,34 & 0,22\end{array}$

$\begin{array}{lcc}\text { P6 } & \text { P7 } & \text { P8 } \\ 0,55 & 0,35 & 0,56 \\ \text { P6 } & \text { P7 } & \text { P8 } \\ 0,92 & 0,52 & 0,88 \\ \text { P6 } & \text { P7 } & \text { P8 } \\ 0,51 & 0,57 & 0,45\end{array}$

Figura 2. Variáveis selecionadas dos funcionários

Na Figura 2, a variável sexo com valor 0 significa masculino e 1 feminino. A variável casado com valor 1 significa que o funcionário é casado e com o valor 0 não é casado.

Não consideramos o problema como uma série temporal mas, dado que a produtividade passada poderá ter correlação com a produtividade futura, criaram-se 8 variáveis com os valores da produtividade nas 8 semanas anteriores à previsão. As variáveis de $P 1$ a $P 8$ referem-se portanto a observações da produtividade do passado de cada individuo. Isso significa que $\mathrm{P} 1$ corresponde à produtividade 1 semana antes, P2 à produtividade 2 semanas antes, e assim por diante até $\mathrm{P} 8$ que corresponde à produtividade 8 semanas antes do momento da previsão para a semana seguinte.
Distribuição temporal da informação de entrada A distribuição das características/variáveis foi efetuada conforme a Figura 2. Note-se contudo que esse não é o formato completo para a entrada de dados nos modelos de previsão. O formato integral das entradas para cada indivíduo, considerando o total de 4 semanas anteriores, será conforme a Figura 3.
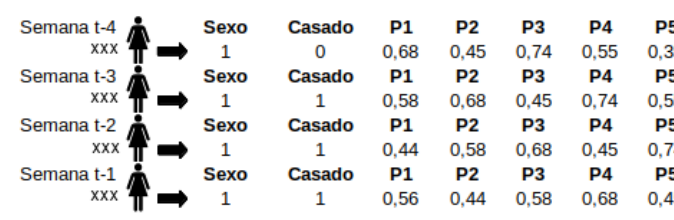

$\begin{array}{cccc}\text { P5 } & \text { P6 } & \text { P7 } & \text { P8 } \\ 0,35 & 0,56 & 0,74 & 0,35 \\ \text { P5 } & \text { P6 } & \text { P7 } & \text { P8 } \\ 0,55 & 0,35 & 0,56 & 0,74 \\ \text { P5 } & \text { P6 } & \text { P7 } & \text { P8 } \\ 0,74 & 0,55 & 0,35 & 0,56 \\ \text { P5 } & \text { P6 } & \text { P7 } & \text { P8 } \\ 0,45 & 0,74 & 0,55 & 0,35\end{array}$

Figura 3. Distribuição temporal da informação da colaboradora xxx

Na Figura 3 vemos os dados da funcionária xxx, contemplando a sua produtividade nas quatro semanas anteriores (cf. t-1 a t-4). Para as entradas no modelo são sempre considerados todos os colaboradores, com a produtividade referente às quatro semanas anteriores de cada um. Como pode ser observado, cada semana por colaborador representa uma linha de dados para entrada.

Fases sequenciais de treino e teste A Figura 3 apresenta o formato de entrada de dados para os modelos. No entanto, antes da previsão, é necessário encontrar a melhor hiper parametrização e selecionar as característica/variáveis de entrada que melhores resultados oferecem, tal como mencionamos nas secções 6 e 7 anteriores. Para estas etapas, os modelos foram avaliados individualmente e, por isso, os dados foram divididos respeitando a técnica Nested Cross Validation Cochrane (2018).

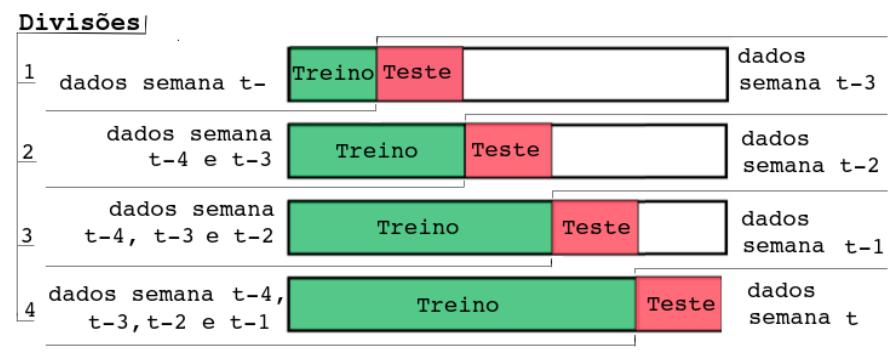

Figura 4. Nested Cross Validation aplicada à previsão de produtividade

A Figura 4 apresenta o procedimento de treino/teste e respectiva organização dos data frames usando a técnica Nested Cross Validation. Nesta técnica os dados são divididos em treino e teste dentro de períodos de tempo, que no presente trabalho corresponde a uma periodicidade semanal representada por t. Os dados de cada período em questão são submetidos aos modelos.

No final do processo, a avaliação de cada modelo efetua-se através da média dos resultados obtidos com a validação das saídas de cada uma das divisões Cochrane (2018). Seguindo critérios do exemplo da secção 8 Figura 8 , os dados são validados pela métricas Area Under the Curve (AUC) Fawcett (2005), Precision Davis and Goadrich (2006), Accuracy Sampson and Babarczy (2003), entre outros.

Para o processo de validação por Nested Cross Validation, utilizado nesse trabalho, considerou-se as 3 primeiras divisões da Figura 4. Os resultados AUC foram registrados 
e calculada uma média dos 3 valores para chegar ao AUC final.

A quarta e última divisão foi reservada como entrada na etapa de previsão, porque usar a mesma divisão no processo de validação geraria Overfitting Srivastava (2014).

Nesta secção exemplificou-se simplificadamente a organização dos data frames recorrendo a apenas três instâncias de funcionários. Na realidade a quantidade de funcionários e as variáveis de entrada são muito mais numerosas. No entanto todo o processo segue exatamente o mesmo procedimento apresentado, em particular nas variáveis que transportam a produtividade das oito semanas anteriores.

\subsection{Normalização dos dados}

A normalização dos valores dos dados foi efetuada para harmonizar as escalas das variáveis de entrada nos modelos. O processo corresponde numericamente à adaptação que é feita para diminuir distâncias muito díspares Gonçalves (2014), uniformizando proporcionalmente todos os valores contínuos para o intervalo entre 0 e 1 .

\subsection{Binarização}

Utilizou-se também um processo de binarização que, para cada valor distinto de uma variável categórica, cria uma variável binária Rajalaxmi and Natarajan (2008). Por exemplo, para re-codificar a variável nominal estado civil, foram criadas três variáveis binárias, i.e., uma para cada categoria identificada na Figura 5. Desta forma, para uma pessoa que tenha o estado civil casado, a variável binária casado recebe o valor 1 , enquanto que as duas variáveis binárias restantes recebem o valor 0 .

\begin{tabular}{|c|c|c|c|}
\hline & Pessoa & Estado Civil & \multirow{2}{*}{$\underset{\pi}{\infty} \rightarrow \mathrm{xxx}$} \\
\hline & $\frac{\mathrm{xxx}}{\mathrm{xxx}}$ & Casado & \\
\hline & yyy & Casado & $\rightarrow$ yуy \\
\hline & \multirow[t]{2}{*}{$\mathrm{zzZ}$} & Divorciado & \\
\hline & & Variável co & inária \\
\hline Pessoa & Solteiro & Casado & Divorciado \\
\hline $\mathrm{xxx}$ & 0 & 1 & 0 \\
\hline yyy & 0 & 1 & 0 \\
\hline ZZZ & 0 & 0 & 1 \\
\hline
\end{tabular}

Figura 5. Conversão de variável categórica para binária

\section{MODELOS DE CLASSIFICAÇÃO UTILIZADOS}

Os modelos utilizados e testados neste trabalho são descritos brevemente a seguir.

Random Forest (RF) é um algoritmo de classificação supervisionada e seu conceito é baseado num conjunto de árvores de decisão Breiman (2001).

Multilayer Perceptron (MLP) é um algoritmo supervisionado e um tipo comum de redes de neurônios dispostos em camadas que possuem conexões unidirecionais entre eles Sanchis (1991).

Naive Bayes (NB) é um dos algoritmos de classificação mais simples, entre os modelos convencionais, na medida em que assume que todos os atributos são independentes uns dos outros, dado o contexto da classe N. Friedman and Goldszmidt (1997).

Extreme Gradient Boost (XGBoost) é um algoritmo com escalabilidade do processo. O sistema funciona mais rápido do que as soluções populares existentes, com condições de recursos e memória limitadas. Chen and Guestrin (2016).

Logistic Regression (LR) é um modelo básico de classificação, dos mais utilizados para predição com variáveis binárias ou categóricas dependentes Rohit and Ajit (2016).

Dummy Classifier (DMM) é um classificador que faz previsões usando regras simples para comparar com outros classificadores.

\section{ALGORITMO GENÉTICO PARA SELEÇÃO}

A utilização de um algoritmo genético foi uma das estratégias utilizadas na seleção das variáveis de entrada para os modelos de previsão. Como é comum na utilização deste tipo de recurso, definiu-se qual faixa de cruzamento, taxa de mutação, número de indivíduos na população e número de gerações.

A estrutura genérica do algoritmo genético aplicado às etapas de hiper parametrização e seleção de variáveis pode ser visualizado na Figura 6. Neste trabalho, o algoritmo é iniciado com a criação de uma população de 15 indivíduos e finalizado quando atingido o critério de parada de 10 gerações. A faixa de cruzamento foi de $50 \%$ e a taxa de mutação 2\% Lee (1993).

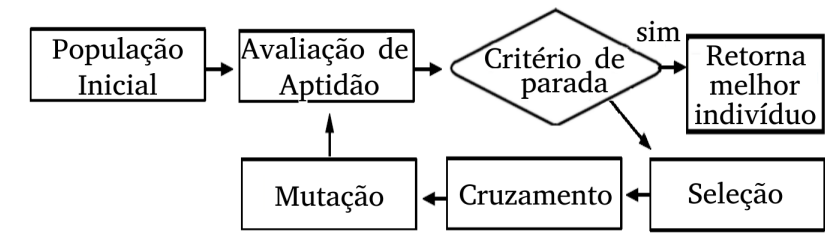

Figura 6. Algoritmo Genético de Seleção

No exemplo da Figura 7 são apresentados 4 indivíduos (A1, A2, A3 e A4) que se submetem ao processo de recombinação genética (cruzamento). A aplicação deste processo na etapa de hiper parametrização lida com valores contínuos em seus genes, enquanto que para a etapa de seleção de variáveis os valores são binários. O exemplo da Figura 7 mostra valores binários para os genes.

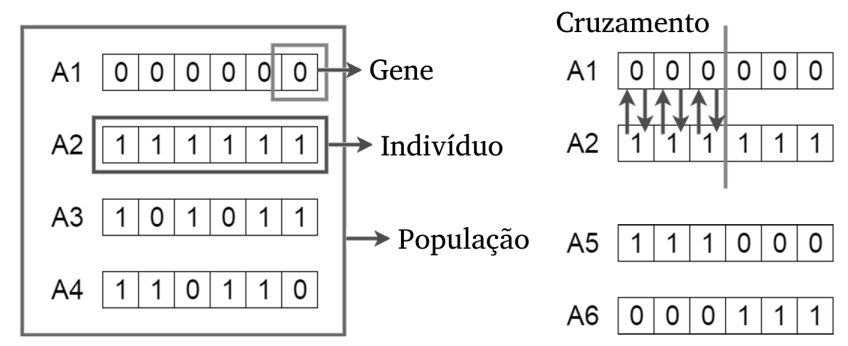

Figura 7. Cruzamento no Algoritmo Genético

A mutação é uma etapa de troca aleatória de valores dos genes, dependendo da taxa de mutação e da avaliação da aptidão do indivíduo, que para este trabalho respeita o que foi apresentado na secção 3.1.3. 


\section{HIPER PARAMETRIZAÇÃO}

A otimização dos valores dos hiper parâmetros dos modelos preditivos é um problema complexo que está além dos limites da capacidade de cálculo humano Smit and Eiben (2009). Utilizar combinações de hiper parâmetros para calibrar os modelos (tuning) pode melhorar os resultados, mas cada caso tem sua especificidade conforme a base de informações utilizada.

\subsection{Hiper Parametrização com Algoritmo Genético}

Neste trabalho, utilizou-se um algoritmo genético para tuning dos modelos. Nessa estratégia, um conjunto de hiper parâmetros definem um indivíduo da população, correspondendo cada um dos parâmetros a um gene.

A função de aptidão dos indivíduo é dada pelos valores gerados, quando os modelos de previsão são executados. O valor mais alto determina o melhor indivíduo e, consequentemente, os melhores parâmetros. O processo de avaliação do indivíduo segue o que foi explicado na seção 3.1.3.

\section{SELEÇÃO DE VARIÁVEIS}

\subsection{Seleção com Correlação de Pearson}

Entre muitos métodos de análise de correlação, na prática, o coeficiente de correlação de Pearson (PPMCC) talvez seja a medida de correlação mais comum na estatística Mari and Kotz (2001). A correlação entre os conjuntos de dados é uma medida que informa o quão próximos eles estão relacionados. No caso deste trabalho, a correlação é feita para ordenar as variáveis mais correlacionadas com a variável de saída (produtividade).

\subsection{Seleção com Backward}

Na seleção de variáveis pelo backward, o conjunto inicial de variáveis é integral, indo-se excluindo cada variável, da menos correlacionada para a mais correlacionada, conforme a correlação de Pearson Abe (2005). Após isso, é feita a submissão dos dados ao modelo. Se o resultado de previsão, avaliado como descrito na secção 3.1.3, for maior que o resultado obtido com a variável antes da sua exclusão, a variável tem sua exclusão confirmada. Caso o resultado seja menor do que o valor obtido com a variável antes da sua exclusão, então a variável é re-integrada no conjunto inicial.

O conjunto de variáveis selecionadas nesta etapa se torna um indivíduo da primeira geração do algoritmo genético de seleção de variáveis.

\subsection{Seleção com Algoritmo Genético}

Na seleção de variáveis, utilizando o algoritmo genético deste trabalho, o indivíduo da população é o conjunto de todas as variáveis de entrada, sendo cada uma delas um gene. Cada gene pode ter um valor de 1 ou 0 , indicando se a variável irá ou não ser utilizada no modelo de previsão. No final do processo, o indivíduo com maior pontuação é escolhido e terá as variáveis selecionadas pelo algoritmo genético. $\mathrm{O}$ processo de avaliação do indivíduo segue $\mathrm{O}$ procedimento descrito na secção 3.1.3.
Tabela 1. Tempo (hh:mm:ss) de processamento dos modelos por etapa

\begin{tabular}{|l|r|r|r|r|}
\hline \multirow{2}{*}{$2^{*}$ Modelos } & $\begin{array}{c}\text { Hiper } \\
\text { Parame- } \\
\text { trização }\end{array}$ & \multicolumn{3}{|c|}{ Seleção de Variáveis } \\
\cline { 2 - 5 } & $\begin{array}{c}\text { Algoritmo } \\
\text { Genético }\end{array}$ & Backward & $\begin{array}{c}\text { Algoritmo } \\
\text { Genético }\end{array}$ & N $^{\circ}$ Vars \\
\hline DMM & $00: 07: 31$ & $01: 24: 00$ & $00: 07: 35$ & 236 \\
\hline NB & $00: 03: 47$ & $01: 40: 03$ & $00: 05: 22$ & 107 \\
\hline RF & $21: 31: 17$ & $\begin{array}{r}16 \text { dias, } \\
18: 25: 07\end{array}$ & $\begin{array}{r}7 \text { dias, } \\
15: 39: 57\end{array}$ & 365 \\
\hline LR & $02: 59: 23$ & $16: 35: 17$ & $02: 57: 46$ & 308 \\
\hline MLP & $04: 08: 37$ & $\begin{array}{r}2 \text { dias, } \\
3: 46: 07\end{array}$ & $04: 41: 03$ & 368 \\
\hline XGBoost & $08: 45: 23$ & $\begin{array}{r}1 \text { dia, } \\
12: 07: 58\end{array}$ & $01: 02: 03$ & 118 \\
\hline
\end{tabular}

Tabela 2. Resultados da previsão de produtividade

\begin{tabular}{|l|l|l|l|l|}
\hline Modelos & AUC & ACCURACY & PRECISION & N $^{\mathbf{O}}$ Vars \\
\hline DMM & 0,50 & 0,51 & 0,51 & 236 \\
\hline NB & 0,71 & 0,66 & 0,67 & 107 \\
\hline RF & 0,83 & 0,75 & 0,73 & 103 \\
\hline LR & 0,75 & 0,69 & 0,70 & 308 \\
\hline MLP & 0,79 & 0,72 & 0,73 & 368 \\
\hline XGBoost & 0,80 & 0,73 & 0,74 & 118 \\
\hline
\end{tabular}

\section{PREVISÃO DE PRODUTIVIDADE}

O processo de treino e teste dos modelos utilizados na previsão de produtividade segue a metodologia de Nested Cross Validation apresentada na seção 3.1.3. Aquando da previsão, os dados da semana t-4, t-3, t-2 e t-1 são utilizados como treino e os dados da semana t como teste. A produtividade é depois estimada pela saída do modelo correspondente à semana $t+1$.

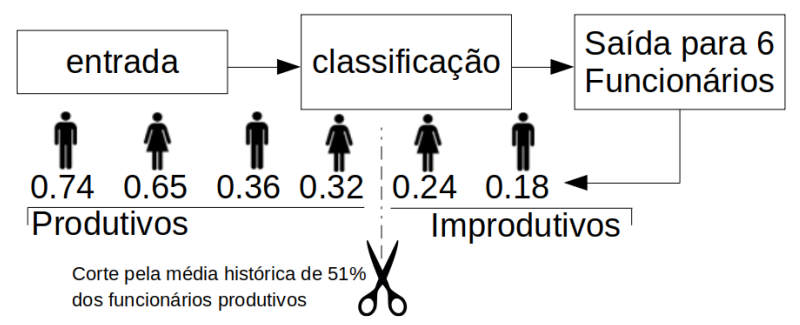

Figura 8. Determinação de funcionários classificados como produtivos

A classificação da produtividade para cada funcionário corresponde a um valor entre 0 e 1 relacionado à proximidade com as classes. Nesse contexto, a saída próxima a 0 é a classe improdutivo e 1 a classe produtivo.

Para se determinar acima de qual valor de proximidade com a classe produtivo os funcionários são classificados, efetua-se um corte na lista ordenada por valor de saída. Esse corte baseia-se na média dos produtivos no histórico de 90 dias da empresa, que gira em torno de $51 \%$ dos funcionários. Nota-se no exemplo da Figura 8.

A Tabela 1 mostra o tempo de execução das etapas, em hh:mm:ss, conforme a arquitetura apresentada na Figura 1.

A Tabela 2 mostra os resultados obtidos na previsão da produtividade em função das métricas previstas (cf. $A U C$, Precision, Accuracy) e de acordo com as etapas definidas na arquitetura da Figura 1. 


\section{CONCLUSÃO}

Este trabalho permitiu descrever, implementar e analisar as peculiaridades do problema de previsão de produtividade de colaboradores de uma grande empresa de teleatendimento. Foi necessário verificar e consultar regras de legislação e obter aprovação dos órgãos éticos, para podermos coletar os dados dos funcionários, cumprindo todos os preceitos legais e regras deontológicas.

Neste trabalho foram utilizados vários modelos de previsão com potencial de aplicação na previsão da produtividade de funcionários de teleatendimento. Estes modelos foram avaliados em comparação com modelos ingênuos NB e DMM. Os modelos que não conseguiram gerar resultados dentro da faixa de previsão de uma semana (i.e. 7 dias), acabaram sendo descartados.

A análise dos modelos avaliados permitiu escolher o modelo que mais se adaptou ao problema de previsão de produtividade, considerando como critérios: o menor tempo de execução e a qualidade dos resultados de previsão obtidos.

A análise dos resultados obtidos a partir dos modelos testados, permite concluir que o problema de previsão de produtividade através do algoritmo XGBoost, demostrou resultados mais interessantes, tendo sido este o algoritmo selecionado pelo tempo de execução. $\mathrm{O}$ algoritmo RF também chamou muito a atenção, sendo um algoritmo baseado em árvore de decisão como o XGBoost Chen and Guestrin (2016)Breiman (2001).

A solução adotada propõe uma arquitetura genérica que tendo sido avaliada em cenários concretos, permitiu à empresa adotar procedimentos de gestão baseados nas previsões efetuadas. Realça-se ainda que este trabalho, em particular a arquitetura proposta e os algoritmos de seleção de hiper-parametrização e seleção de variáveis de entrada nos algoritmos de classificação, tem um enorme potencial de aplicação noutros processos de previsão da empresa (e.g. absenteísmo), podendo deste modo contribuir positivamente para o seu processo de gestão e decisão.

\section{REFERÊNCIAS}

Abe, S. (2005). Modified Backward Feature Selection by Cross Validation. European Symposium on Artificial Neural Networks, 27-29.

Breiman, L. (2001). Random Forest. Kluwer Academic Publishers. Manufactured in The Netherlands. Machine Learning, 45, 5-32.

Chen, T. and Guestrin, C. (2016). XGBoost: A Scalable Tree Boosting System. Proceedings of the 22nd ACM SIGKDD International Conference on Knowledge Discovery and Data Mining Pages 785-794.

Cochrane, C. (2018). Time Series Nested CrossValidation. Towards Data Science/acessado 24/03/2019: https://towardsdatascience.com/time-series-nestedcross-validation-76adba623eb9.

Davis, J. and Goadrich, M. (2006). The Relationship Beteween Precision-Recall and ROC Curvesy. Technical Report - University of Wisconsin - Madison.

Fawcett, T. (2005). An introduction to ROC analysis. Institute for the Study of Learning and Expertise.

Gonçalves, C.D.F. (2014). Gestão da Manutenção em Edifícios:Modelos para uma abordagem LARG. Tese -
Faculdade de Ciência e Tecnologia - Universidade Nova Lisboa.

Lee, T. (1993). Dynamic control of genetic algorithms using fuzzy logic techniques. S. Forrest, Ed., Proc. of the Fifh Int. Conf. on Genetic Algorithms.

Mari, D. and Kotz, S. (2001). Correlation and Dependence. London: Imperial College Press.

N. Friedman, D.G. and Goldszmidt, M. (1997). Bayesian network classifiers. Machine Learning, 29:131-163.

Rajalaxmi, R.R. and Natarajan, A.M. (2008). An Effective Data Transformation Approach for Privacy Preserving Clustering. Article in Journal of Computer Science 4 (4): $320-326$.

Rohit, P. and Ajit, P. (2016). Prediction of Employee Turnover in Organizations using Machine Learning Algorithms A case for Extreme Gradient Boosting. International Journal of Advanced Research in Artificial Intelligence, Vol. 5, No. 9.

Sampson, G. and Babarczy, A. (2003). A Test of the LeafAncestor Metric for Parse Accuracy. School of Cognitive and Computing Sciences - University of Sussex.

Sanchis, E. (1991). Learning Structural Models of Subword units through Grammatical Inference Techniques. ICASSP-91 Proc. pages 189-192.

Silva, A.M. (2007). Condições De Trabalho E Adoecimento Dos Trabalhadores Em Teleatendimento: Uma Breve Revisão. Revista de Gestão Integrada em Saúde do Trabalho e Meio Ambiente - v.1, n.3, Artigo 7.

Smit, S. and Eiben, A. (2009). Comparing parameter tuning methods for evolutionary algorithms. CEC'09. IEEE Congress.

Srivastava, N. (2014). Dropout: A Simple Way to Prevent Neural Networks from Overfitting. Journal of Machine Learning Research 15.

Ávila Assunção, A. and de Oliveira Vilela, L.V. (2003). As condições de adoecimento em uma empresa de teleatendimento. Editora Faculdade de Medicina UFMG. 